\title{
A derivation of the optimal answer-copying index and some applications*
}

\author{
Mauricio Romero ${ }^{\dagger \ddagger} \quad$ Álvaro Riascos ${ }^{\S \ddagger} \quad$ Diego Jara ${ }^{\ddagger}$
}

February 20, 2018

\begin{abstract}
Multiple-choice exams are frequently used as an efficient and objective method to assess learning but they are more vulnerable to answer-copying than tests based on open questions. Several statistical tests (known as indices in the literature) have been proposed to detect cheating; however, to the best of our knowledge they all lack mathematical support that guarantees optimality in any sense. We partially fill this void by deriving the uniform most powerful (UMP) under the assumption that the response distribution is known. In practice, however, we must estimate a behavioral model that yields a response distribution for each question. We calculate the empirical type-I and type-II error rates for several indices that assume different behavioral models using simulations based on real data from twelve nationwide multiple-choice exams taken by 5 th and 9th graders in Colombia. We find that the index with the highest power among those studied, subject to the restriction of preserving the type-I error, is one based on the work of Wollack (1997) and Linden and Sotaridona 2006) and is
\end{abstract}

*Corresponding author: Mauricio Romero. e-mail: mtromero@ucsd.edu The authors would like to thank the ICFES for financial support, three anonymous referees, Nicola Persico, Decio Coviello and Julian Mariño and his group of statisticians for valuable comments and suggestions.

${ }^{\dagger}$ University of California - San Diego.

‡Quantil | Matemáticas Aplicadas.

$\S$ Universidad de los Andes. 
superior to the indices studied and developed by Wesolowsky (2000) and Frary, Tideman, and Watts (1977). We compare the results of applying this index to all 12 exams and find that examination rooms with stricter proctoring have a lower level of copying. Finally, a Bonferroni correction to control for the false positive rate is proposed to detect massive cheating.

Key Words: $\omega$ Index, Answer Copying, False Discovery Rate, NeymanPearson's Lemma.

JEL Clasification: C19, I20

\section{Introduction}

Multiple-choice exams are frequently used as an efficient and objective way of evaluating knowledge. Nevertheless, they are more vulnerable to answer copying than tests based on open questions. Answer-copy indices provide a statistical tool for detecting cheating by examining suspiciously similar response patterns between two students. However, these indices have three problems. First, similar answer patterns between a pair of students could be justified without answer copying. For example, two individuals with very similar educational background are likely to provide similar answers. The second problem is that a statistical test (an index) is by no means a conclusive basis for accusing someone of copying, since it is impossible to completely eliminate type-I errors. In other words, it is possible that two individuals share the same response pattern by chance. Finally, every index assumes responses are stochastic. If the assumed probability distribution is incorrect, the index can lead to incorrect conclusions. Furthermore, all the indices in the literature are ad-hoc and there are no theoretical results that support the use of one index over the other.

Wollack (2003) compares several indices and finds that among those that preserve size the $\omega$ index is the most powerful one. However, the set of indices studied is not comprehensive and in particular does not include the index 
developed by Wesolowsky (2000).

Thus there are two gaps in the literature that this article seeks to fill. First, it provides theoretical foundations that validate the use of indices that reject the null hypothesis of no cheating for a large number of identical answers under the assumption that student responses are stochastic.

Second, it compares the type-I and type-II error rates of the $\omega$ and $\gamma$ indices for answer copy detection, based on the work of Wollack 1997 11 and Wesolowsky (2000) respectively ${ }^{2}$ Using Monte Carlo simulations and data from the SABER tests taken by 5th and 9th graders in Colombia in May and October of 2009 we find that the conditional version of the standardized index first developed by Wollack (1997) is the most powerful among those that respect size.

We compare the results of applying the index to examination rooms with different strategies to control cheating. We find a negative correlation between the level of proctoring and the prevalence of copying. We also find a lower prevalence of copying in examination rooms where students answer different portions of the test at the same time compared to examination rooms where all students answer the same portion of the test at the same time. These results have at least two possible interpretations: they could be interpreted as evidence that the index is indeed detecting cheating, or, alternatively, if one believes that the index can be used as a reliable measure of cheating, these results can be interpreted as estimates of how effective current strategies for cheating-prevention are. However, the results of these two exercises must be taken cautiously as they are not the result of a randomized experiment and therefore might be biased due to unobservable factors.

Our article has a fourth contribution. We outline a procedure for detecting massive cheating. These indices detect individual cheating, but do not consti-

\footnotetext{
${ }^{1}$ In this article we use a version closer to the work of Linden and Sotaridona (2006).

${ }^{2}$ Both indices are refinements of the indices first developed by Frary et al. (1977).
} 
tute definitive proof of copying given their statistical nature. They are merely intended to raise flags. In Colombia an index is used to search for examination rooms with a large number of flags (i.e. a large proportion of students guilty of copying according to the index). When a large number of flags are raised, every student in the examination room must retake the test under stricter surveillance conditions. The appropriate way to search for a large number of flags is to test multiple hypothesis at the same time, but these procedures often results in low statistical power. We apply a Bonferroni correction outlined by Benjamini and Hochberg (1995) to detect multiple cheating while controlling for the false positive rate. The application is straightforward and we think this is a useful tool for flagging possible examination rooms where massive cheating might have occurred. This information could be used, as in Colombia, to make entire examination rooms retake an exam under stricter surveillance conditions.

The article is organized as follows. The second section derives an optimal statistical test (index) to detect answer copying using the Neyman-Pearson's Lemma. The third section presents two of the most widely used indices, which are based on the work of Wollack (1997), Frary et al. (1977), Wesolowsky (2000), and Sotaridona, van der Linden, and Meijer (2006). The fourth section presents a brief summary of the data used and is followed by a section that presents the methodology of the Monte Carlo simulations used to find the empirical type-I and type-II error rates (to test which behavioral model gives the best results) and its results. Section six analyses the correlation between different strategies to control cheating and the prevalence of cheating according to the index and section seven presents the results of using a Bonferroni correction to calculate the prevalence of massive cheating. Finally the last section concludes. 


\section{Applying Neyman-Pearson's to answer copy- ing}

It is normal for two answer patterns to have similarities by chance. Answercopying indices try to detect similarities that are so unlikely to happen naturally that answer-copying becomes a more natural explanation than chance. Most answer-copy indices are calculated by counting the number of identical answers between the test taker suspected of copying and the test taker suspected of providing answer: 3 . In all these indices the null hypothesis is the same: there is no cheating.

All these indices are ad-hoc since they are not derived to be optimal in any sense. To the extent of the authors' knowledge, this article presents the first effort to rationalize the use of these indices to detect answer copying using the Neyman-Pearson's Lemma (NPL) (Neyman \& Pearson, 1933) resulting in the uniformly most powerful (UMP) test (index), assuming we know the underlying probability of responses for each individual in each question. However, we must turn to empirical data to find the performance of each index since different behavioral models result in different response distributions.

First, let us state the problem formally. Let us assume that there are $N$ questions and $n$ alternatives for each question. We are interested in testing whether the individual who cheated (denoted by $c$ ) copied from the individual who supposedly provided the answers (denoted by $s$ ). Let $\gamma_{c s}$ be the number of

\footnotetext{
${ }^{3}$ For examples see Linden and Sotaridona 2004,2006$)$; Sotaridona and Meijer $(2003$, 2002); Sotaridona et al. (2006); Holland (1996); Frary et al. (1977); Cohen (1960); Bellezza and Bellezza (1989); Angoff (1974); Wesolowsky 2000); Wollack (1997)
} 
questions that $c$ copied from $s$. The objective is to test the following hypotheses:

$$
\begin{aligned}
& H_{0}: \gamma_{c s}=0 \\
& H_{1}: \gamma_{c s}>0
\end{aligned}
$$

Let $I_{c s i}$ be equal to one when individuals $c$ and $s$ have the same answer to question $i$ and zero otherwise. Then, the number of common answers between $c$ and $s$ can be expressed as:

$$
M_{c s}=\sum_{i=1}^{N} I_{c s i}
$$

Under the null hypothesis $M_{c s}$ is the sum of $N$ independent Bernoulli random variables, each with a different probability of success $\pi_{i}$, equal to the probability that individual $c$ has the same answer as individual $s$ in question $i$. The distribution of $M_{c s}$ is known as a poisson binomial distribution. Let $B\left(\pi_{1}, \ldots, \pi_{N}\right)$ be such distribution and $f_{N}\left(x ; \pi_{1}, \ldots, \pi_{N}\right)$ the probability mass function (pmf) at $x$. Notice that if $\pi_{1}=\pi_{2}=\ldots=\pi_{N}=\pi$ then the poisson binomial distribution reduces to a standard binomial distribution.

Now, let $A$ denote the set of questions that student $c$ copied from $s$. Then if $|A|=k$, it means that $\gamma_{c s}=k$, and $M_{c s}$ has the following probability mass function (pmf) $\hat{f}_{N}\left(x ; \pi_{1}, \ldots, \pi_{N}, A\right)$, where we define $\hat{f}_{N}\left(x ; \pi_{1}, \ldots, \pi_{N}, A\right) \doteq$ $f_{N}\left(x, \pi_{1}^{\prime}, . ., \pi_{N}^{\prime}\right)$ such that

$$
\pi_{i}^{\prime}= \begin{cases}1 & \text { if } i \in A \\ \pi_{i} & \text { if } i \notin A\end{cases}
$$

For example, say that there are 50 questions and that the students copied 
questions 1,10 and 50, i.e. $A=\{1,10,50\}$ then

$$
\hat{f}_{N}\left(x ; \pi_{1}, \ldots, \pi_{N}, A\right)=f_{N}\left(x ; 1, \pi_{2}, \ldots, \pi_{9}, 1, \pi_{11}, \ldots, \pi_{49}, 1\right) .
$$

Before we continue let us state Neyman-Pearson's Lemma (NPL):

Theorem 1. Neyman-Pearson's Lemma (Casella \& Berger, 2002)

Consider testing $H_{0}: \theta=\theta_{0}$ against $H_{1}: \theta=\theta_{1}$ where the pmf is $f\left(\boldsymbol{x} \mid \theta_{i}\right)$, $i=0,1$, using a statistical test (index) with rejection region $R$ that satisfies

$$
\begin{gathered}
\boldsymbol{x} \in R \text { if } f\left(x \mid \theta_{1}\right)>f\left(x \mid \theta_{0}\right) k \\
\boldsymbol{x} \in R^{c} \text { if } f\left(x \mid \theta_{1}\right)<f\left(x \mid \theta_{0}\right) k
\end{gathered}
$$

for some $k \geq 0$, and

$$
\alpha=P_{H_{0}}(\boldsymbol{X} \in R)
$$

Then

1. (Sufficiency) Any test (index) that satisfies equations 2 and 3 is a UMP level $\alpha$ test (index).

2. (Necessity) If there exists a test (index) satisfying equations 2 and 3 with $k>0$, then every UMP level $\alpha$ test (index) is a size $\alpha$ test (index) satisfies 3 - and every UMP level $\alpha$ test (index) satisfies 2 except perhaps on a set $A$ such that $P_{H_{0}}(\boldsymbol{X} \in A)=P_{H_{1}}(\boldsymbol{X} \in A)=0$.

the test (index) is the uniformly most powerful (UMP) level $\alpha$ test (index).

In this context, let us apply the NPL to the simple hypothesis test $H_{0}: A=$ $A_{0}$ and $H_{1}: A=A_{1}$, where $A_{0}=\emptyset$ (i.e. there is no cheating) and $A_{1}$ is a set of questions, to get the UMP test. If in the data we observe $x$ questions answered 
equally by individuals $c$ and $s$ then the likelihood ratio test $t^{4}$ would be:

$$
\lambda^{A}(x)=\frac{\hat{f}_{N}\left(x ; \pi_{1}, \ldots, \pi_{N}, A\right)}{f_{N}\left(x ; \pi_{1}, \ldots, \pi_{N}\right)}
$$

Now we must find the critical value of the test. In other words, we need the greatest value $c$ such that under the null we have:

$$
1-P_{H_{0}}\left(\frac{\hat{f}_{N}\left(x ; \pi_{1}, \ldots, \pi_{N}, A\right)}{f\left(x ; \pi_{1}, \ldots, \pi_{N}\right)}<c\right)=P_{H_{0}}\left(\frac{\hat{f}_{N}\left(x ; \pi_{1}, \ldots, \pi_{N}, A\right)}{f_{N}\left(x ; \pi_{1}, \ldots, \pi_{N}\right)}>c\right) \leq \alpha
$$

For any given pair of simple hypotheses $\left(H_{0}: A=A_{0}, H_{1}: A=A_{1}\right)$ we know how to find the UMP (by using the NPL). The following lemma will allow us to find the UMP for more complex alternative hypothesis (e.g. $H_{1}:\{A$ : $|A| \geq 1\})$.

Lemma 1. $\lambda^{A}(x)=\frac{\hat{f}_{N}\left(x ; \pi_{1}, \ldots, \pi_{N}, A\right)}{f_{N}\left(x, \pi_{1}, \ldots, \pi_{N}\right)}$ is increasing in $x \in\{0, \ldots, N\}$ for all $A$.

Before we present the proof we must first recall some useful results proved by Wang (1993).

Theorem 2 (Theorem 2 in Wang (1993)). The pmf of a poisson binomial satisfies the following inequality:

$$
f_{N}\left(x ; \pi_{1}, \pi_{2}, \ldots, \pi_{N}\right)^{2}>C(x) f_{N}\left(x+1 ; \pi_{1}, \pi_{2}, \ldots, \pi_{N}\right) f_{N}\left(x-1 ; \pi_{1}, \pi_{2}, \ldots, \pi_{N}\right)
$$

where $C(x)=\max \left(\frac{x+1}{x}, \frac{N-x+1}{N-x}\right)$

which has as an immediate corollary

Corollary 1. The pmf of a poisson binomial satisfies the following inequality:

$$
f_{N}\left(x ; \pi_{1}, \pi_{2}, \ldots, \pi_{N}\right)^{2} \geq f_{N}\left(x+1 ; \pi_{1}, \pi_{2}, \ldots, \pi_{N}\right) f_{N}\left(x-1 ; \pi_{1}, \pi_{2}, \ldots, \pi_{N}\right)
$$

\footnotetext{
${ }^{4}$ Notice that NPL implies that a likelihood ratio test is the uniformly most powerful (UMP) test for simple hypothesis testing.
} 
Now we are ready to prove the lemma:

Proof of Lemma 1. We consider the case $|A|=1$, given that the proof for the case when $|A|>1$ can be obtained by induction. Without loss of generality, assume $A=\{1\}$. The numerator in the lemma's quotient is 0 for $x=0$, so we proceed to prove monotonicity $\lambda^{A}(x)$ in $x$ for $x \geq 1$. Likewise, the case $N=1$ follows trivially, so we assume $N>1$.

For simplicity, we call $g(x)=f_{N-1}\left(x ; \pi_{2}, \ldots, \pi_{N}\right)$. First, note that

$$
\hat{f}_{N}\left(x ; \pi_{1}, \ldots, \pi_{N} ; A\right)=g(x-1) .
$$

Second, corollary 1 states that $g(x-1) g(x+1) \leq g(x)^{2}$. Third, we can write $f_{N}\left(x ; \pi_{2}, \ldots, \pi_{N}\right)=\pi_{1} g(x-1)+\left(1-\pi_{1}\right) g(x)$. With these observations we have

$$
\begin{aligned}
\frac{\hat{f}_{N}\left(x ; \pi_{1}, \ldots, \pi_{N} ; A\right)}{f_{N}\left(x ; \pi_{1}, \ldots, \pi_{N}\right)} & =\frac{g(x-1)}{\pi_{1} g(x-1)+\left(1-\pi_{1}\right) g(x)} \times \frac{\pi_{1} g(x)+\left(1-\pi_{1}\right) g(x+1)}{\pi_{1} g(x)+\left(1-\pi_{1}\right) g(x+1)} \\
& \leq \frac{\pi_{1} g(x) g(x-1)+\left(1-\pi_{1}\right) g(x)^{2}}{\left[\pi_{1} g(x-1)+\left(1-\pi_{1}\right) g(x)\right]\left[\pi_{1} g(x)+\left(1-\pi_{1}\right) g(x+1)\right]} \\
& =\frac{g(x)}{\pi_{1} g(x)+\left(1-\pi_{1}\right) g(x+1)} \\
& =\frac{\hat{f}_{N}\left(x+1 ; \pi_{1}, \ldots, \pi_{N} ; A\right)}{f_{N}\left(x+1 ; \pi_{1}, \ldots, \pi_{N}\right)}
\end{aligned}
$$

Given that $\frac{\hat{f}_{N}\left(x ; \pi_{1}, \ldots, \pi_{N} ; A\right)}{f_{N}\left(x ; \pi_{1}, \ldots, \pi_{N}\right)}$ is increasing in $x$ for all $A$ then we have that for every $c$ there exists a $k^{*}$ such that $P_{H_{0}}\left(\frac{\hat{f}_{N}\left(x ; \pi_{1}, \ldots, \pi_{N}, A\right)}{f_{N}\left(x ; \pi_{1}, \ldots, \pi_{N}\right)}<c\right)=\sum_{w=0}^{k^{*}} f_{N}\left(w, \pi_{1}, \ldots, \pi_{N}\right)$.

In particular for a given level $\alpha$ of the test we can find $k^{*}$ such that

$$
1-P_{H_{0}}\left(\frac{\hat{f}_{N}\left(x ; \pi_{1}, \ldots, \pi_{N}, A\right)}{f\left(x ; \pi_{1}, \ldots, \pi_{N}\right)}<c\right)=\sum_{w=0}^{k^{*}} f\left(w, \pi_{1}, \ldots, \pi_{N}\right) \leq \alpha
$$

Then, if we reject the null hypothesis when $M_{c s}>k^{*}$, we get the UMP for 
a particular set A. However, the rejection region is the same for all $A$, thus if we reject the null hypothesis when $M_{c s}>k^{*}$, we get the UMP for all $A$ such that $|A| \geq 1$. This justifies the use of indices that reject the null hypothesis for large values of $M_{c s}$.

However, $\pi_{i}$ must be estimated somehow (it was taken as known in this section and thus in empirical applications we do not have the UMP) and different methods yield different results. We now turn to the data to find out which index performs better in practice.

Frary et al. (1977) in a seminal article developed the first indices, known as $g_{1}$ and $g_{2}$, that reject the null hypothesis for large values of $M_{c s}$. Wollack (1997), Linden and Sotaridona (2006) and Wesolowsky (2000) have proposed further refinements of Frary et al. (1977) methods. We will evaluate the performance of these indices in practice.

\section{Copy Indices}

Let us assume that student $j$ has a probability $\pi_{i v}^{j}$ of answering option $v$ on question $i$. The probability that two students have the same answer on question $i\left(\pi_{i}\right)$ can be calculated in two ways. First, assuming independent answers, the probability of obtaining the same answer is $\pi_{i}=\sum_{v=1}^{n} \pi_{i v}^{c} \pi_{i v}^{s}$.

Second, we could think of the answers of individual $s$ as being fixed, as if he were the source of the answers and $c$ the student who copies. In the absence of cheating, conditional on the answers of $s$, the probability that individual $c$ has the same answer as individual $s$ in question $i$ is $\pi_{i}=\pi_{i v_{s}}^{c}$, where $\pi_{i v_{s}}^{c}$ is the probability that individual $c$ answered option $v_{s}$ which was chosen by $s$ in question $i$.

A discussion of these two approaches is given in Frary et al. (1977) and Linden and Sotaridona (2006). The first is known as the unconditional index 
and is symmetric in the sense that the choice of who is $s$ and who is $c$ is irrelevant since $\pi_{i}$ is the same either way. The second is known as the conditional index and it is not symmetric opening the possibility that the index rejects the null hypothesis that student $a$ copied from student $b$ but not rejecting the null hypothesis that $b$ copied from $a$. The details of each situation determine which approach is appropriate. If we believe students copied from each other or answered the test jointly then a conditional index is undesirable, but if we believe that a student is the source (for whatever reason) of answers but did not collaborate with the cheater, then a conditional index might be more appropriate. We study both conditional and unconditional indices.

Indices vary along three dimensions. The first dimension is how they estimate $\pi_{i v}^{j}$. The second is whether they are a conditional or an unconditional index. Finally, they vary how critical values are calculated. They either use the exact distribution (a poisson binomial distribution) or a normal distribution, by applying some version of the central limit theorem.

In order to use the central limit theorem in this context recall $M_{c s}$ is the sum of $N$ Bernoulli variables and has mean $\sum_{i=1}^{N} \pi_{i}$ and variance $\sum_{i=1}^{N} \pi_{i}\left(1-\pi_{i}\right)$. Thus $\frac{M_{c s}-\sum_{i=1}^{N} \pi_{i}}{\sqrt{\sum_{i=1}^{N} \pi_{i}\left(1-\pi_{i}\right)}}$ converges in distribution to a standard normal distribution as $N$ goes to infinity. There are two advantages to the normal approximation. First critical values are easier to calculate and more precise (computationally) and second it allows for a finer choice of critical values.

As mentioned before, Frary et al. (1977) developed the first indices, known as $g_{1}$ and $g_{2}$, that reject the null hypothesis for large values of $M_{c s}$. However, both Wesolowsky (2000) and Wollack (2003) show that variations of the original method proposed by Frary et al. (1977) yield superior results, and in this article we study the indices they developed. The first variation is the $\omega$ index developed by Wollack (1997) that assumes there is an underlying nominal response model. 
The second variation is the $\gamma$ index developed by Wesolowsky (2000) based on a variation of Frary et al. (1977) work.

\section{$3.1 \omega$ index}

The $\omega$ index is based on the work of Wollack (1997) and assumes a nominal response model that allows the probability of answering a given option to vary across questions and individuals. As before, let $N$ be the number of questions and $n$ the number of alternatives for answering each question. Suppose that an individual with skill $\theta_{j}$, who does not copy, responds with probability $\pi_{i v}$ for option $v$ to question $i$. In other words:

$$
\pi_{i v}\left(\theta_{j}\right)=\frac{e^{\xi_{i v}+\lambda_{i v} \theta_{j}}}{\sum_{h=1}^{m} e^{\xi_{i h}+\lambda_{i h} \theta_{j}}},
$$

where $\xi_{i v}$ y $\lambda_{i v}$ are model parameters and are known as the intercept and slope, respectively. The intercept and slope can vary across questions. The parameters of the questions $\left(\xi_{i v}\right.$ and $\left.\lambda_{i v}\right)$ are estimated using marginal maximum likelihood, while ability is estimated using the EAP method (Expected A Posteriori). The estimation is performed using the rirt package in $\mathrm{R}$ (Germain, Abdous, \& Valois, 2014 5 . It is necessary to estimate ability as the proportion of correct answers taking into account that a correct answer to a "difficult" question indicates a higher ability than a correct answer to a "simple" question. More information on marginal maximum likelihood and EAP can be found in Linden and Hambleton (1997) and Hambleton, Swaminathan, and Rogers (1991).

Let $\omega_{1}$ and $\omega_{2}$ be the unconditional and conditional (exact) versions of this index (following somewhat the $g_{1}$ and $g_{2}$ notation of Frary et al. (1977)) and let $\omega_{1}^{s}$ and $\omega_{2}^{s}$ be the standardized versions (i.e. they use the normal distribution to find the critical values of the index).

\footnotetext{
${ }^{5}$ The package rirt can be found on: http://libirt.sourceforge.net/
} 


\section{$3.2 \gamma$ index}

The indices developed by Wesolowsky (2000), which are an extension and improvement of the indices developed by Frary et al. (1977), assume that the probability that student $j$ has the correct answer in question $i$ is given by:

$$
p_{i}=\left(1-\left(1-r_{i}\right)^{a_{j}}\right)^{1 / a_{j}},
$$

where $r_{i}$ is the proportion of students that had the right answer in question $i$. The parameter $a_{j}$ is estimated by solving the equations

$$
\frac{\sum_{i=1}^{N} p_{i}}{n}=c_{j}
$$

where $c_{j}$ is the proportion of questions answered correctly by individual $j$. Finally, we need the probability that student $j$ chooses option $v$ among those that are incorrect which is estimated as the proportion of students with an incorrect answer that chose each incorrect option. Lets denote $\gamma_{1}$ and $\gamma_{2}$ the unconditional and conditional version of this index and by $\gamma_{1}^{s}$ and $\gamma_{2}^{s}$ their standardized version respectively.

Before we compare how the different versions of the $\omega$ and the $\gamma$ index fare in practice, the following section presents the data that will be used.

\section{Data}

In Colombia, every student enrolled in 5th, 9 th or 11th grade, whether attending a private or a public school, has to take a standardized multiple-choice test known as the SABER test ${ }^{6}$. These exams are intended to measure the performance of students and schools across several areas. The Instituto Colombiano

\footnotetext{
${ }^{6}$ The tests in the 5 th and 9 th grade have been somewhat irregular and with students being tested every 2 to 3 years.
} 
para la Evaluación de la Educación (ICFES) is in charge of developing, distributing and applying these exams. The score of the 11th grade test is used by most universities in Colombia as an admission criterion. The ICFES also evaluates all university students during their senior year. We analyze the 5th and 9th grade tests for 2009. In total, we have 12 different exams depending on the subject, the date of the exam and the grade of the student 7 The following abbreviations, used by the ICFES, are used: per grade, 50 for 5th and 90 for 9th. Per area, 041 for mathematics, 042 for language and 043 for science. Per date, F1 for May and F2 for October. For example, exam PBA9041F2 is taken by 9 th graders for mathematics in October. A brief overview of each test is presented in Table 1.

For each exam the database contains the answer to each question for each individual, as well as the examination room where the exam was taken. The correct answers for each exam are also available.

Table 1: Summary statistics

\begin{tabular}{|l|l|l|l|l|l|l|}
\hline Test & Subject & Grade & Month & Questions & Students & $\begin{array}{l}\text { Examination } \\
\text { Rooms }\end{array}$ \\
\hline PBA5041F1 & Math & 5th & May & 48 & 60,099 & 3,421 \\
PBA5041F2 & Math & 5th & Oct & 48 & 403,624 & 31,827 \\
PBA5042F1 & Language & 5th & May & 36 & 60,455 & 3,441 \\
PBA5042F2 & Language & 5th & Oct & 36 & 402,508 & 31,642 \\
PBA5043F1 & Science & 5th & May & 48 & 60,404 & 3,432 \\
PBA5043F2 & Science & 5th & Oct & 48 & 405,537 & 31,833 \\
PBA9041F1 & Math & 9th & May & 54 & 44,577 & 1,110 \\
PBA9041F2 & Math & 9th & Oct & 54 & 303,233 & 9,059 \\
PBA9042F1 & Language & 9th & May & 54 & 44,876 & 1,110 \\
PBA9042F2 & Language & 9th & Oct & 54 & 302,781 & 9,044 \\
PBA9043F1 & Science & 9th & May & 54 & 44,820 & 1,107 \\
PBA9043F2 & Science & 9th & Oct & 54 & 30,3723 & 9,053 \\
\hline
\end{tabular}

Source: ICFES. Calculations: Authors.

\footnotetext{
${ }^{7}$ Each grade (5th and 9th) presents three tests: Science, Mathematics and Language. Schools that finish the academic year in December present the exam in September and schools that finish their academic year in June present the exam in May. In total there are two dates, two grades and three subjects, for a total of 12 exams.
} 


\section{Index Comparison}

In this section we compare the different versions of the $\omega$ and the $\gamma$ indeces. In order to do this we evaluate the type-I and type-II error rates by creating synthetic samples in which we control the level of cheating between individuals.

\subsection{Methodology}

To find the empirical type-I error rate, individuals who could not have possibly copied from one another are paired together and tested for cheating using a particular index. This is done by pairing individuals that answered the exam in different examination rooms, thus eliminating the possibility of answer copying. The empirical type-I error rate is calculated as the proportion of pairs for which the index rejects the null hypothesis. To find the empirical type-II error rate, we take these answer-copy free pairs and simulate copying by forcing specific answers to be the same. The proportion of pairs for which the index rejects the null hypothesis is the power of the index 8

To make things clearer, let $c$ denote the test taker suspected of cheating, $s$ the test taker believed to have served as the source of answers. The steps taken to find the type-I error rate and the power of each index are as follows:

1. 100,000 pairs are picked in such a way that for each couple the individuals performed the exam in different examination rooms.

2. The answer-copy methodology is applied to these pairs, and the proportion of pairs for which the index rejects the null hypothesis is the empirical type-I error rate estimator.

3. To calculate the power of the index, the answer pattern for individual $c$ is

\footnotetext{
${ }^{8}$ Recall that the power of the test is the complement of the type-II error rate, i.e. Power $=$ $100 \%$ - TypeIIError.
} 
changed by replacing $k$ of his answers to match to those of individual $s 9$

(a) The level of copy, $k$, is set, and is defined as the number of answers transferred from $s$ to $c$.

(b) $k$ questions are selected randomly.

(c) Individual c's answers for the $k$ questions are changed to replicate exactly those of individual $s$. Answers that were originally identical count as part of the $k$ questions being changed.

4. We apply the answer-copy methodology to the pairs whose exams have been altered. The proportion of pairs accused of cheating is the power of the index for a copying level of $k$.

\section{$5.2 \quad$ Results}

Throughout the analysis a confidence level $(\alpha)$ of $99.9 \%$ is used and the power of the index is calculated at copying levels $(k)$ of: $1,5,10,15,20, \ldots, N$, where $N$ is the number of questions in the exam.

\subsubsection{Type-I error rate}

As can be seen in Tables 2 and 3 the $\gamma_{2}, \gamma_{2}^{s}$, and the $\omega_{2}$ indices have an empirical type-I error rate that is consistently above the theoretical type-I error rate of one in a thousand. The $\gamma_{1}$ index (developed by Wesolowsky (2000)) empirical error rate is above the theoretical one in several cases.

Based on these results, we discard the $\gamma_{2}, \gamma_{2}^{s}$ and the $\omega_{2}$ indices and restrict the search for the most powerful index among $\gamma_{1}, \gamma_{1}^{s}, \omega_{1}$ and $\omega_{2}^{s}$.

\footnotetext{
${ }^{9}$ For example, let us assume the answer pattern for $s$ is $A C B C D A D C D A B$, which means that there were 11 questions and that he/she answered $\mathrm{A}$ for the first questions, $\mathrm{C}$ for the second questions, and so on. Also assume that the original answer pattern of $c$ without copying is $D C A B C D A A B C B$. Let $k$ be 5 , this means and let us assume that the randomly selected questions were $1,4,5,10,11$. This means that the modified (with copying) answer patterns for $c$ will be $A C A C D D A A B A B$.
} 
Table 2: Type-I error rate for the $\gamma$ indices

\begin{tabular}{|c|c|c|c|c|c|c|c|}
\hline Exam & Subject & Grade & Month & $\gamma_{1}$ & $\gamma_{2}$ & $\gamma_{1}^{s}$ & $\gamma_{2}^{s}$ \\
\hline PBA5041F1 & Mathematics & 5 th & May & 0.66 & 2.20 & 0.41 & 0.76 \\
PBA5041F2 & Mathematics & 5 th & October & 0.87 & 2.44 & 0.59 & 1.11 \\
PBA5042F1 & Language & 5th & May & 1.20 & 2.18 & 0.77 & 1.16 \\
PBA5042F2 & Language & 5th & October & 1.21 & 2.36 & 0.92 & 1.49 \\
PBA5043F1 & Science & 5 th & May & 1.05 & 2.59 & 0.73 & 1.38 \\
PBA5043F2 & Science & 5th & October & 0.74 & 1.81 & 0.61 & 1.24 \\
PBA9041F1 & Mathematics & 9th & May & 1.38 & 1.97 & 0.96 & 1.26 \\
PBA9041F2 & Mathematics & 9th & October & 2.15 & 2.14 & 1.69 & 1.53 \\
PBA9042F1 & Language & 9th & May & 0.85 & 2.24 & 0.56 & 1.04 \\
PBA9042F2 & Language & 9 th & October & 0.84 & 1.92 & 0.59 & 1.34 \\
PBA9043F1 & Science & 9th & May & 1.32 & 2.06 & 0.93 & 1.42 \\
PBA9043F2 & Science & 9th & October & 1.02 & 1.70 & 0.74 & 1.37 \\
\hline
\end{tabular}

Source: ICFES. Calculations: Authors.

Number of copy-free couples accused of copying (for every 1,000 pairs)

at a $99.9 \%$ confidence level

Table 3: Type-I error rate for the $\omega$ indices

\begin{tabular}{|c|c|c|c|c|c|c|c|}
\hline Exam & Subject & Grade & Month & $\omega_{1}$ & $\omega_{2}$ & $\omega_{1}^{s}$ & $\omega_{2}^{s}$ \\
\hline PBA5041F1 & Mathematics & 5th & May & 0.42 & 1.28 & 0.23 & 0.52 \\
PBA5041F2 & Mathematics & 5 th & October & 0.61 & 1.38 & 0.31 & 0.78 \\
PBA5042F1 & Language & 5th & May & 0.80 & 1.61 & 0.46 & 0.73 \\
PBA5042F2 & Language & 5th & October & 0.86 & 1.51 & 0.55 & 0.95 \\
PBA5043F1 & Science & 5 th & May & 0.79 & 1.37 & 0.47 & 0.87 \\
PBA5043F2 & Science & 5th & October & 0.82 & 1.47 & 0.57 & 0.88 \\
PBA9041F1 & Mathematics & 9th & May & 0.89 & 1.53 & 0.58 & 0.89 \\
PBA9041F2 & Mathematics & 9th & October & 1.22 & 1.53 & 0.99 & 1.07 \\
PBA9042F1 & Language & 9th & May & 0.55 & 1.44 & 0.31 & 0.65 \\
PBA9042F2 & Language & 9th & October & 0.86 & 1.47 & 0.63 & 0.97 \\
PBA9043F1 & Science & 9th & May & 0.78 & 1.46 & 0.59 & 0.98 \\
PBA9043F2 & Science & 9th & October & 0.78 & 1.36 & 0.63 & 1.03 \\
\hline
\end{tabular}

Source: ICFES. Calculations: Authors.

Number of copy-free couples accused of copying (for every 1,000 pairs)

at a $99.9 \%$ confidence level

\subsubsection{Power}

The following figures show the power among the $\gamma_{1}, \gamma_{1}^{s}, \omega_{1}$ and $\omega_{2}^{s}$ indices in the Mathematics 5th grade May test. Notice that the $\omega_{2}^{s}$ index has the highest power for all levels of answer copying. This is true for all exams as shown in 
figures 5.15 in appendix $\mathrm{A}$. Based on the results of the previous section and this section, we believe this justifies the use of the $\omega_{2}^{s}$ index over all otther version of the $\omega$ index and all versions of the $\gamma$ index.

In other words, the index with the highest power among those studied, subject to the restriction of preserving the type-I error, uses a nominal response model for item answering, conditions the probability of identical answers on the answer pattern of the individual that provides answers, and calculates critical values via a normal approximation.

In the next section we apply the $\omega_{2}^{s}$ to our data and compare the prevalence of cheating across examination rooms in which different strategies to prevent cheating are used by the ICFES.

Figure 1

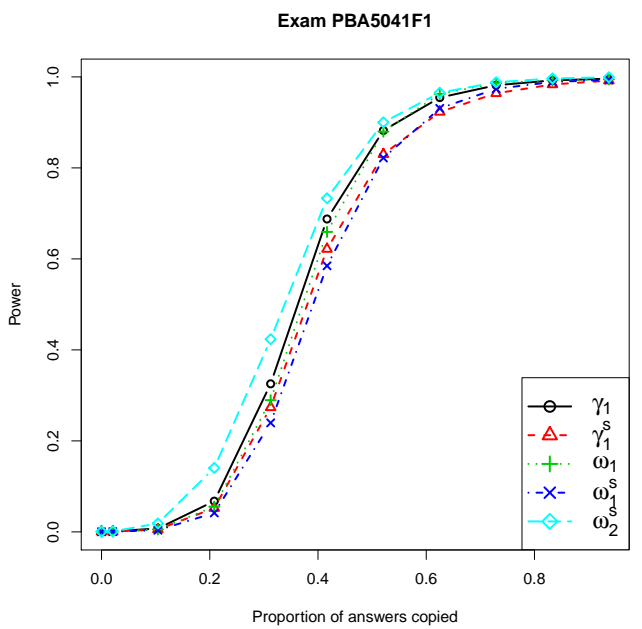

Power in terms of the proportion of answers copied, for all the indices, in the Mathematics 5th grade May test. 


\section{$6 \quad$ Strategies to prevent cheating}

The ICFES randomly assigns schools to three different samples that have different levels of proctoring. Most of the schools are assigned to the censal sample in which the ICFES distributes the exams to the schools and the schools perform the proctoring. The controlada and estadistica samples are smaller but proctoring is done by the government itself. In the controlada sample the proctoring is done by the central government (i.e. the ICFES) while in estadistica sample proctoring is carried out by the regional government (Secretarias de Educación).

Table 4 shows some descriptive statistics of the samples. There are two things that are worth mentioning here. First, in the controlada and estadistica samples the ICFES had different versions of each test, so that each student was randomly assigned to one of three possible tests per subject. We only have information for the students that answered the version of the test that was used in the censal sample. Thus one would expect the average number of students per school in the controlada and estadistica samples to be around one third of those in the censal sample; however, this is not the case. Second, the October estadistica sample has more students per school than either the controlada or the censal samples. These two results lead us to believe that the assignment of schools to samples was not entirely random.

We apply the $\omega_{2}^{s}$ index to the three different samples (see figure 2). In most cases the prevalence of cheating according to the index is lower for the controlada or the estadistica sample and highest for the censal sample. In most cases the controlada sample has a lower prevalence of cheating or a similar level to the estadistica sample, except for the May 9th grade Mathematics (PBA9041F1) test 10

These results can be interpreted in at least two different ways. If one remains

\footnotetext{
${ }^{10}$ This could be due to sampling variation given that there are only 75 schools in the controlada sample.
} 
skeptical about the index then this would serve as evidence that the index is indeed detecting cheating. Alternatively, if one believes that the index can be used as a reliable measure of cheating, these results can be interpreted as the amount of cheating that is prevented by increasing the level of proctoring. However, since the assignment of schools to samples does not seem to be random these results must be taken with caution as unobservable factors could bias the results. Additionally, it is impossible to distinguish the effect of proctoring and of having multiple versions of an exam distributed to students.

Table 4: Characteristics of the controlada, estadistica and censal samples

\begin{tabular}{|c|c|c|c|}
\hline & Controlada & Estadistica & Censal \\
\hline \multicolumn{4}{|c|}{ 5th Grade May } \\
\hline No. Students & 1,413 & 7,648 & 60,099 \\
\hline No. of Schools & 141 & 680 & 3,421 \\
\hline Students/School & 10.02 & 11.25 & 17.57 \\
\hline & $(0.88)$ & $(0.47)$ & $(0.46)$ \\
\hline \multicolumn{4}{|c|}{ 5th Grade October } \\
\hline No. Students & 3,830 & 26,393 & 403,624 \\
\hline No. of Schools & 958 & 654 & 31,827 \\
\hline Students/School & 4.00 & 40.36 & 12.68 \\
\hline & $(0.13)$ & $(1.36)$ & $(0.11)$ \\
\hline \multicolumn{4}{|c|}{ 9th Grade May } \\
\hline No. Students & 1,150 & 6,690 & 44,577 \\
\hline No. of Schools & 75 & 351 & 1110 \\
\hline Students/School & 15.33 & 19.06 & 40.16 \\
\hline & $(1.62)$ & $(1.08)$ & $(1.44)$ \\
\hline \multicolumn{4}{|c|}{ 9th Grade October } \\
\hline No. Students & 3,106 & 24,387 & 303,233 \\
\hline No. of Schools & 495 & 487 & 9,059 \\
\hline Students/School & 6.27 & 50.08 & 33.47 \\
\hline & $(0.25)$ & $(1.74)$ & $(0.35)$ \\
\hline
\end{tabular}

Source: ICFES. Calculations: Authors.

Note: Standard error of the mean for the number of students per school in parenthesis. 
Figure 2: Cheating across samples

\section{Proportion of couples accused of copying}

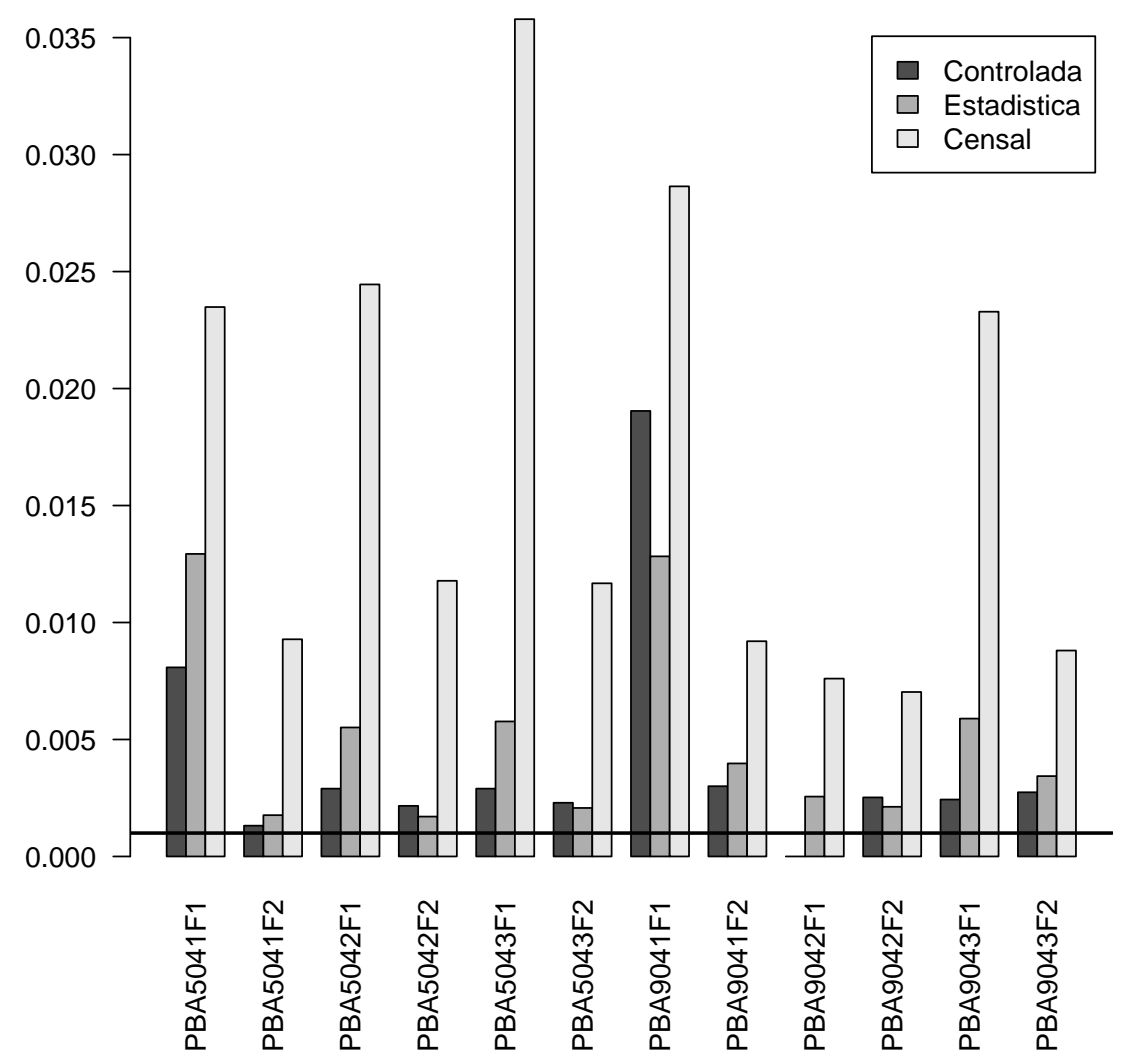

Proportion of couples accused of copying across exams and across samples using the $\omega_{2}^{s}$ index. The horizontal black line is the theoretical type-I error rate. Source: ICFES. Calculations: Authors.

Finally, we restrict ourselves to the censal sample. Figure 3 shows the proportion of couples for which the index rejects the null hypothesis. There is a clear pattern in which cheating drops dramatically between May and October. The SABER tests are administrated over three sessions, wherein students answer a different subject in each session. In May, every student took the same 
subject at the same time, while in October only one third of the students took the same subject in each session, thus reducing the number of students from whom one could copy in a given session. In other words, in May all students took the mathematics portion of the test at the same time. In October, while one third of the students answered the mathematics portion of the test another third answered the language portion and the final third answered the science portion.

Note that the Language Test for 9th graders in May (PBA9042F1 test) does not follow the trend. It is also surprising to find that the levels of cheating are similar for 5th and 9th graders. These populations are different in terms of motivation, maturity and sophistication. We could not find a reasonable explanation for either of these phenomena.

As before, these results can be interpreted in at least two different ways. They could be interpreted as evidence that the index is indeed detecting cheating. Alternatively, if one believes that the index can be used as a reliable measure of cheating, these results can be interpreted as the amount of cheating that is prevented by having different students answer different parts of the exam at different times instead of having all of them answer portion of the exam at the same time. Again this results must be taken with caution as the population of students in May and October might be different in unobservable factors which could bias the results. 
Figure 3

\section{Proportion of couples accused of copying}

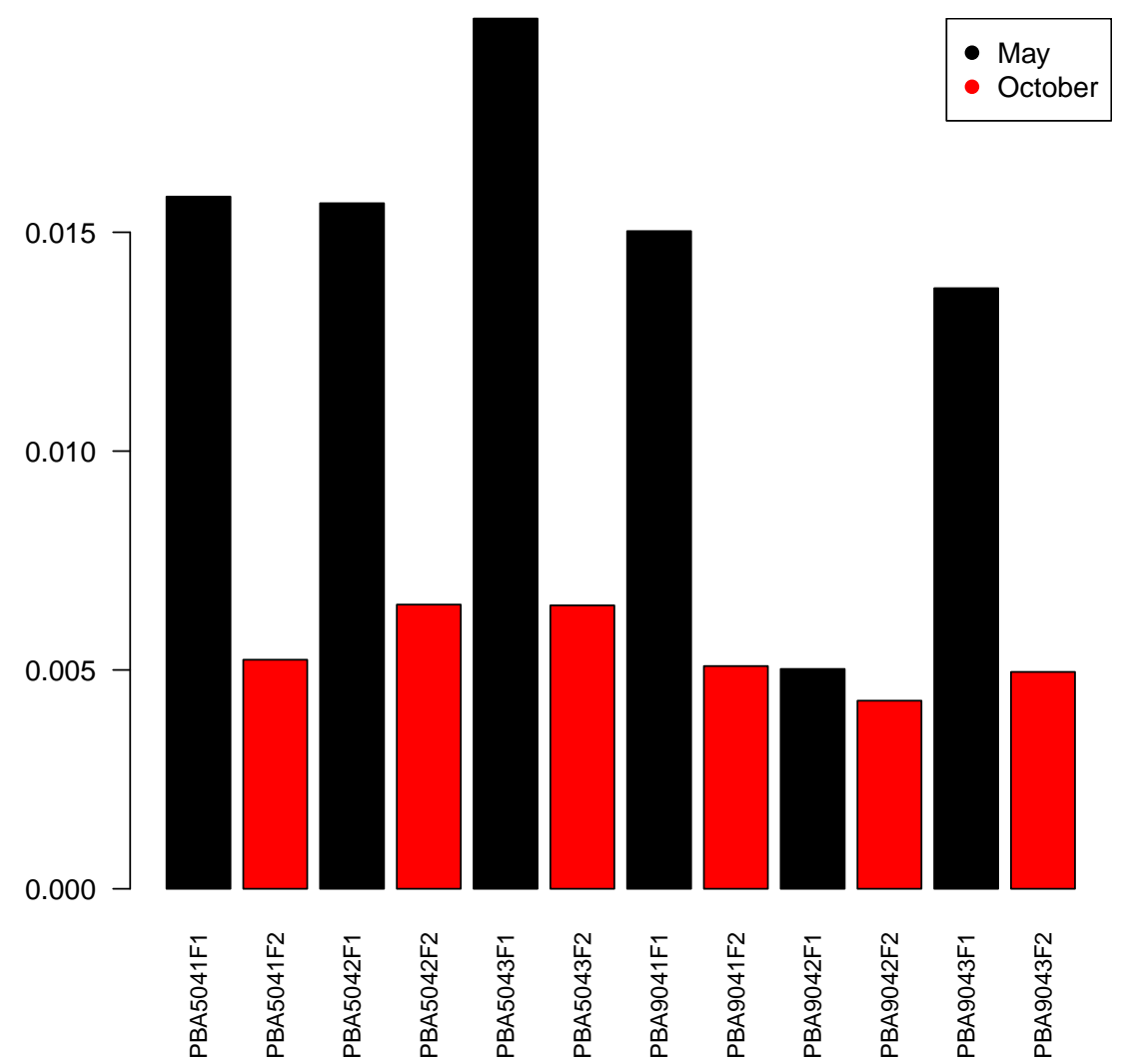

Proportion of couples accused of copying across exams in the censal sample using the $\omega_{2}^{s}$ index. Source: ICFES. Calculations: Authors.

\section{$7 \quad$ Massive cheating}

In this section we consider a subject rarely treated in the answer-copying literature: massive cheating. Many institutions, including the ICFES, do not use answer copying-indices to formally blame an individual of copying. Rather they are interested in detecting highly suspicious examination rooms. The ICFES forces suspicious examination rooms to retake the exam under stricter surveil- 
lance conditions.

To determine whether massive cheating has occurred in an examination room, multiple hypotheses must be tested. If the significance level for a given statistical test is $\alpha_{I}$, the significance level for a multiple test $\left(\alpha_{M T}\right)$ will increase exponentially as the number of hypothesis to be tested increases. In other words, $\alpha_{M T}=1-\left(1-\alpha_{I}\right)^{n} \leq \alpha_{I} \cdot n$, assuming independence across the hypotheses. Thus we need to set $\alpha_{I}=\frac{\alpha_{M T}}{n}$ in order to assure the multiple test significance level; if this correction is made, in most cases the power of the test is severely diminished. To overcome these difficulties a line of research has developed procedures to control error rates similar to the type-I error (of the multiple hypotheses test), which can be easily applied in many cases without compromising the power of the test.

Most of these methodologies are based on Bonferroni correction that control the false positive rate (that is, the number of null hypotheses rejected incorrectly as a proportion of the number of null hypotheses rejected). We use the results from applying the $\omega_{2}^{s}$ index to every examination room. If there are $n$ students in a room then the index is applied $n \times(n-1)$ times and we adjust the p-values following the correction given by Benjamini and Hochberg (1995).

Suppose there are $H_{1}, \ldots, H_{m}$ hypotheses to be tested, ordered such that their $p$-values follow $P_{1} \leq P_{2} \leq \ldots \leq P_{m}$, where $P_{i}$ is the $p$-value of hypothesis $H_{i}$. Let $k$ be the greatest integer $i$, such that:

$$
P_{i} \leq \frac{i}{m} p^{*}
$$

$H_{i}$ is then rejected for every $i \in\{1, \ldots, k\}$. This controls for the false positive rate to a maximum of $p^{*}$ (Benjamini \& Hochberg, 1995). The previous statement is only true if there is independence between true null hypotheses. This assumption implies that the decision to not copy is an individual one and 
is unrelated to the decision not to copy of other individuals. This assumption depends on the conditions under which cheating takes place. For example, if an examination room has poor supervision, it would motivate several students to copy, thus invalidating the assumption.

Figure 4 presents the proportion of examination rooms where more than $60 \% 11$ of the students are suspected of cheating, for $p^{*}=0.01 \%$. As can be seen, there is a high proportion of examination rooms with massive cheating. This could be explained by the fact that the examination rooms consist of students in the same grade in a given school. Nevertheless, the fact that the proportion of massive cheating drops dramatically between May and October is reassuring, since less cheating is expected in the latter. It is also interesting that the levels of massive cheating are lower, in general, for the 9 th $\operatorname{grad} \epsilon^{12}$

\footnotetext{
${ }^{11}$ This is the level used by the ICFES to make students in exmaination rooms retake the test under stricter surveillance conditions.

${ }^{12}$ The ICFES compared our results with information they have regarding school's reputation in terms of "honesty", and found the two to be consistent. Unfortunately, we do not have permission to divulge this information; consequently the results of this comparison cannot be presented here.
} 
Figure 4: Massive cheating per exam

\section{Examination rooms with massive cheating ( $>60 \%$ of students suspected of copying)}

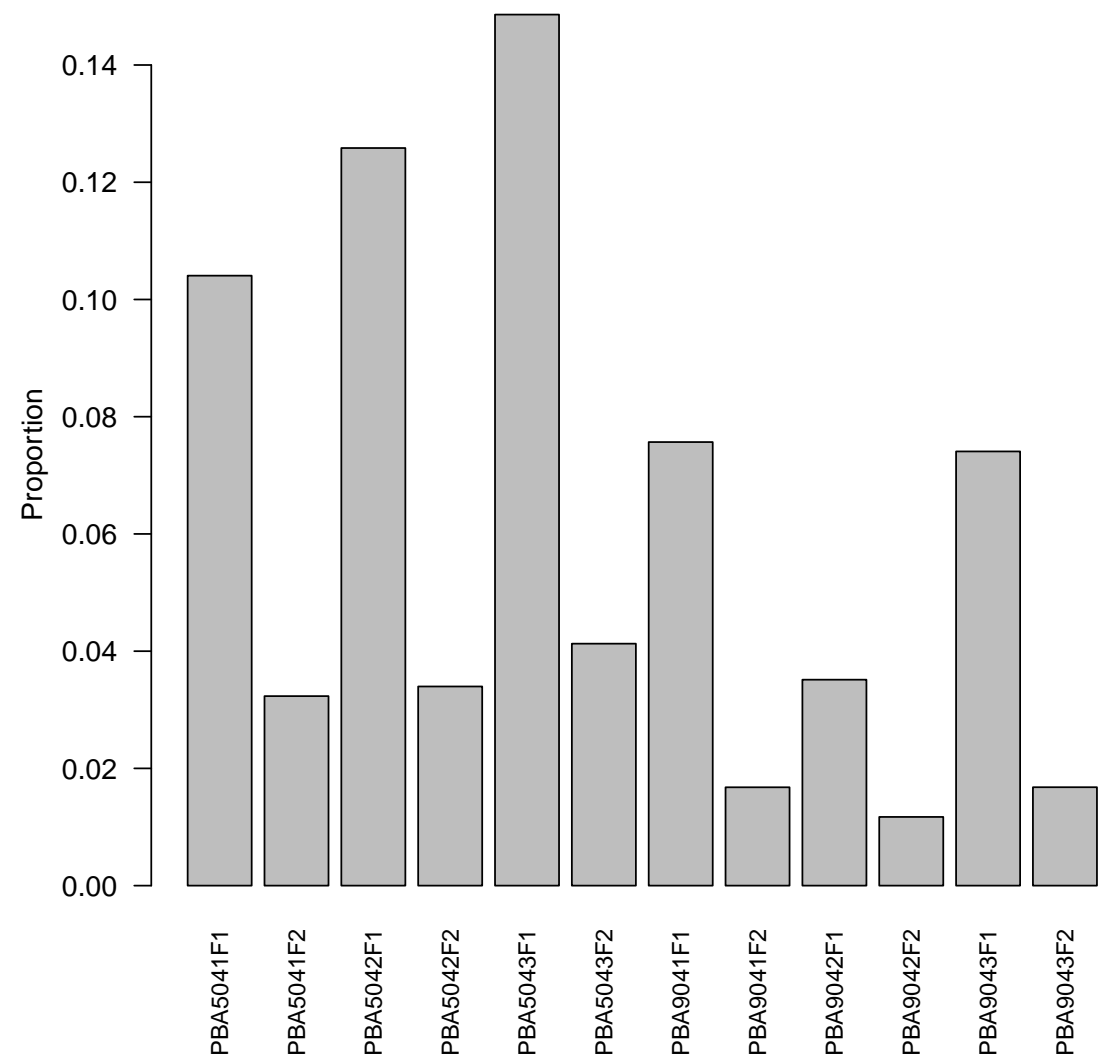

Proportion of examination rooms where more than $60 \%$ of the students are suspected of cheating after using Benjamini and Hochberg (1995) correction for testing multiple hypothesis and controlling the false positive rate at $p^{*}=0.01$. Source: ICFES. Calculations: Authors.

\section{Conclusions}

In this article we justify the use of a variety of statistical tests (known as indices) found in the literature to detect answer copying in standardized tests. We derived the uniform most powerful (UMP) test (index) using the NeymanPearson's Lemma under the assumption that the response distribution is known. 
In practice, a behavioral model for item answering must be estimated and indices vary along which model they assume.

Using data from the SABER 5th and 9th tests taken in May and October of 2009 in Colombia, we compare eight widely used indices that are based on the work of Frary et al. (1977); Wollack (1997); Wesolowsky (2000); Sotaridona et al. (2006). We first filter out the indices that do not meet the theoretical type-I error rate in practice and then select most powerful index among them. The most powerful index, among those that respect the type-I error rate, is a conditional index that models student behavior using a nominal response model, conditions the probability of identical answers on the answer pattern of the individual that provides answers, and relies on the central limit theorem to find critical values.

Using this index we analyze 12 exams taken by 5 th and 9th graders in May and October of 2009 in Colombia. We find a negative correlation between the level of proctoring and the prevalence of cheating. We also find a lower prevalence of copying in examination rooms where students answer different portions of the test at the same time compared to examination rooms where all students answer the same portion of the test at the same time. These results have at least two possible interpretations: they could be interpreted as evidence that the index is indeed detecting cheating, or, alternatively, if one believes that the index can be used as a reliable measure of cheating, these results can be interpreted as the amount of cheating that is prevented by each one of these strategies to control cheating.

Finally, we propose a methodology for detecting massive cheating while controlling for the false positive rate using a Bonferroni correction. Institutions that use answer-copying indices should also use Bonferroni corrections to test for multiple hypothesis as this extension is straightforward. 
We believe the results in this paper should have practical implications and lead to the use of what we call the $\omega_{2}^{s}$ over other indices and the adoption of Bonferroni corrections. Further research should be done to evaluate the effectiveness of different strategies to reduce cheating.

\section{References}

Angoff, W. H. (1974). The development of statistical indices for detecting cheaters. Journal of the American Statistical Association, 69(345), pp. 44-49.

Bellezza, F. S., \& Bellezza, S. F. (1989). Detection of cheating on multiplechoice tests by using error-similarity analysis. Teaching of Psychology, 16(3), pp. 151-155.

Benjamini, Y., \& Hochberg, Y. (1995). Controlling the false discovery rate: A practical and powerful approach to multiple testing. Journal of the Royal Statistical Society. Series B (Methodological), 57(1), pp. 289-300.

Casella, G., \& Berger, R. (2002). Statistical inference. Thomson Learning.

Cohen, J. (1960). A coefficient of agreement for nominal scales. Educational and Psychological Measurement, 20(1), pp. 37-46.

Frary, R. B., Tideman, T. N., \& Watts, T. M. (1977). Indices of cheating on multiple-choice tests. Journal of Educational Statistics, 2(4), pp. 235-256.

Germain, S., Abdous, B., \& Valois, P. (2014). rirt: Item response theory simulation and estimation [Computer software manual]. ( $\mathrm{R}$ package version $1.3 .0)$

Hambleton, R. K., Swaminathan, H., \& Rogers, H. J. (1991). Fundamentals of item response theory. SAGE Publications.

Holland, P. (1996). Assessing unusual agreement between the incorrect answers of two examinees using the $\mathrm{k}$ index: statistical theory and empirial 
support. ETS technical report.

Linden, W. J. v. d., \& Hambleton, R. (1997). Handbook of modern item response theory. Springer.

Linden, W. J. v. d., \& Sotaridona, L. (2004). A statistical test for detecting answer copying on multiple-choice tests. Journal of Educational Measurement, 41(4), pp. 361-377.

Linden, W. J. v. d., \& Sotaridona, L. (2006). Detecting answer copying when the regular response process follows a known response model. Journal of Educational and Behavioral Statistics, $31(3)$, pp. 283-304.

Neyman, J., \& Pearson, E. S. (1933). On the problem of the most efficient tests of statistical hypotheses. Philosophical Transactions of the Royal Society of London. Series A, Containing Papers of a Mathematical or Physical Character, 231, pp. 289-337.

Sotaridona, L. S., \& Meijer, R. R. (2002). Statistical properties of the k-index for detecting answer copying. Journal of Educational Measurement, 39(2), pp. 115-132.

Sotaridona, L. S., \& Meijer, R. R. (2003). Two new statistics to detect answer copying. Journal of Educational Measurement, 40(1), pp. 53-69.

Sotaridona, L. S., van der Linden, W. J., \& Meijer, R. R. (2006). Detecting answer copying using the kappa statistic. Applied Psychological Measurement, $30(5)$, pp. 412-431.

Wang, Y. H. (1993). On the number of successes in independent trials. Statistica Sinica, 3, pp. 295-312.

Wesolowsky, G. (2000). Detecting excessive similarity in answers on multiple choice exams. Journal of Applied Statistics, 27(7), pp. 909-921.

Wollack, J. A. (1997). A nominal response model approach for detecting answer copying. Applied Psychological Measurement, 21 (4), pp. 307-320. 
Wollack, J. A. (2003). Comparison of answer copying indices with real data.

Journal of Educational Measurement, 40(3), pp. 189-205.

\section{A Power}

Figure 5

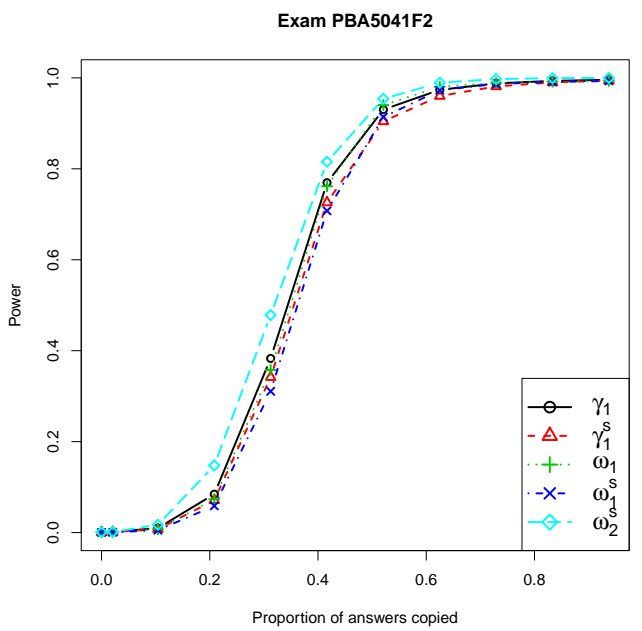

Power in terms of the proportion of answers copied, for all the indices, in the mathematics 5th grade October test. Source: ICFES. Calculations: Authors. 
Figure 6

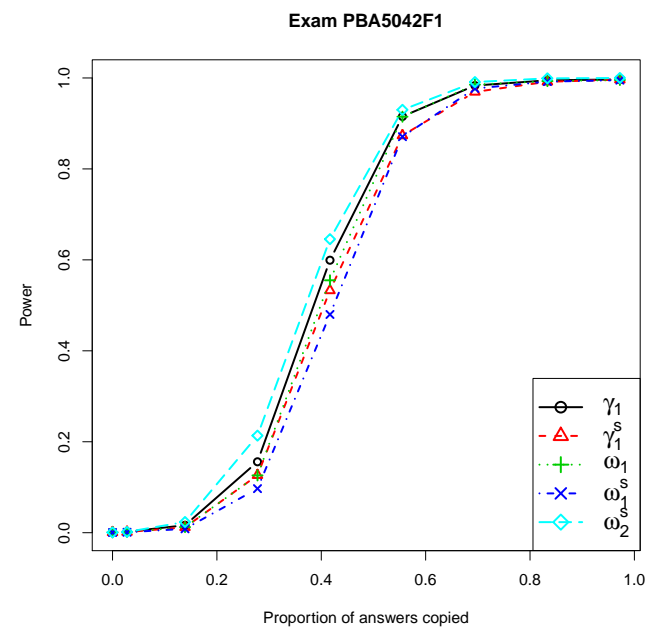

Power in terms of the proportion of answers copied, for all the indices, in the language 5th grade May test. Source: ICFES. Calculations: Authors.

Figure 7

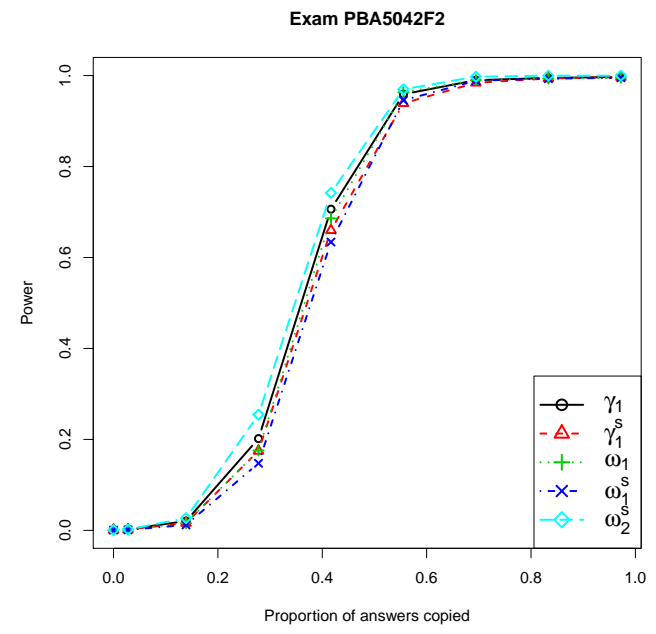

Power in terms of the proportion of answers copied, for all the indices, in the language 5th grade October test. Source: ICFES. Calculations: Authors. 
Figure 8

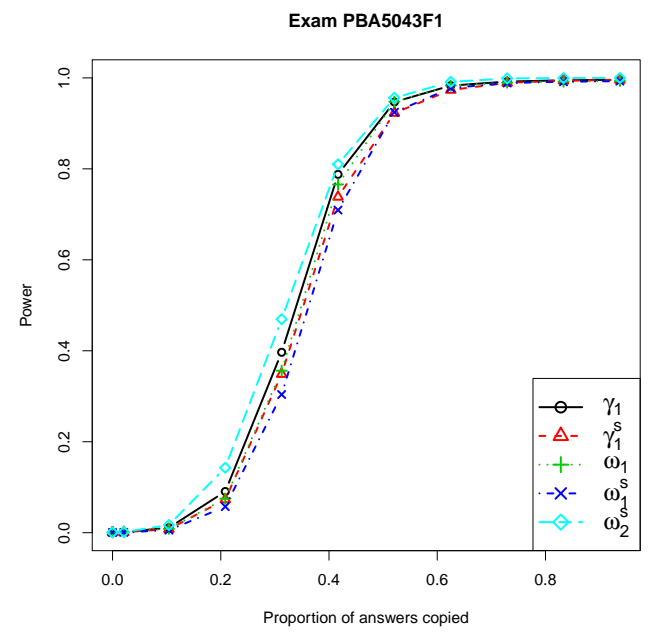

Power in terms of the proportion of answers copied, for all the indices, in the science 5 th grade May test. Source: ICFES. Calculations: Authors.

Figure 9

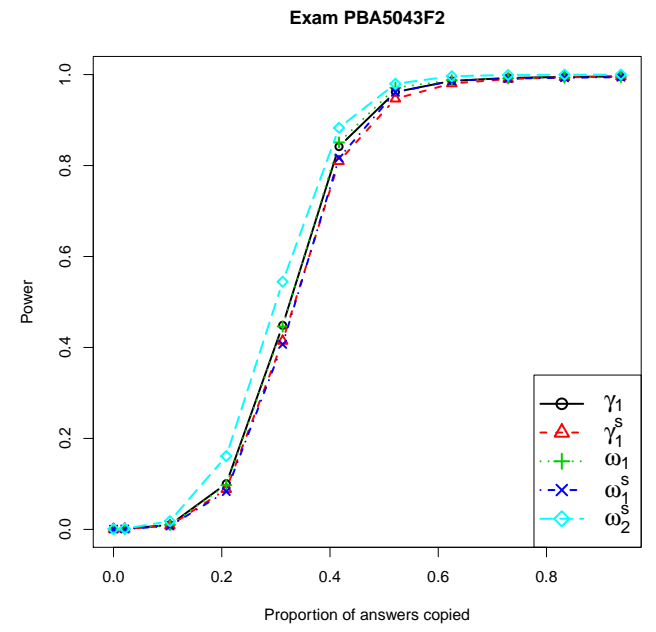

Power in terms of the proportion of answers copied, for all the indices, in the science 5 th grade October test. Source: ICFES. Calculations: Authors. 
Figure 10

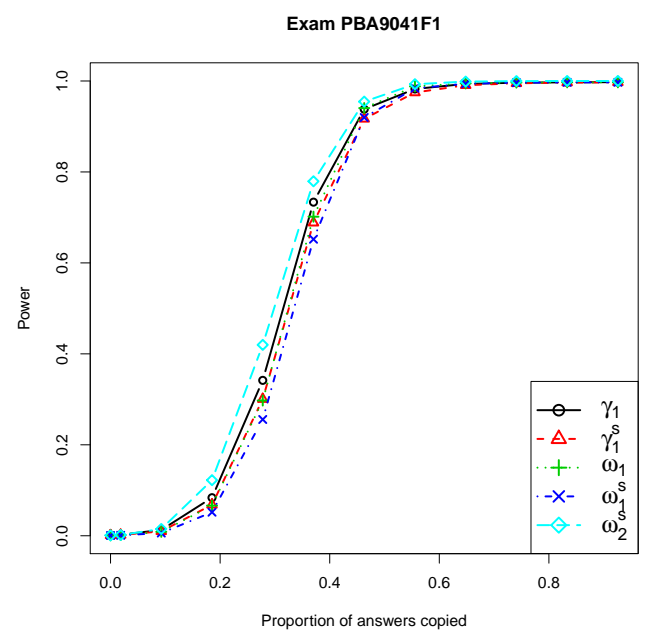

Power in terms of the proportion of answers copied, for all the indices, in the mathematics 9th grade May test. Source: ICFES. Calculations: Authors.

Figure 11

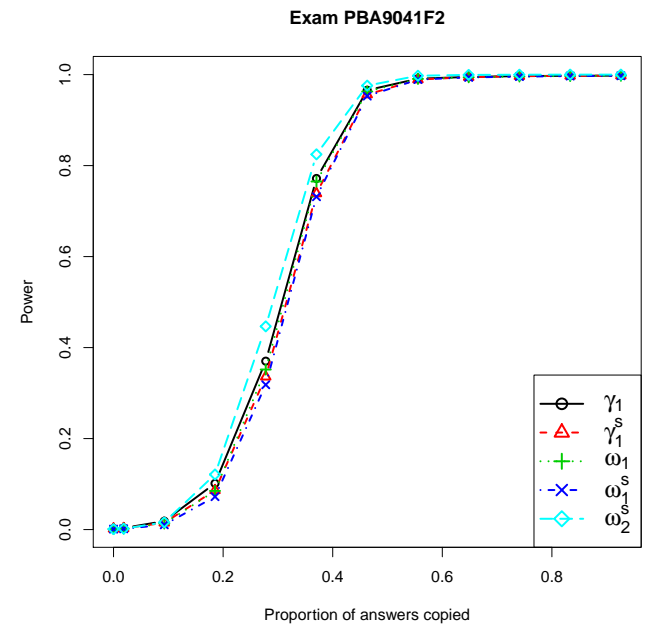

Power in terms of the proportion of answers copied, for all the indices, in the mathematics 9th grade October test. Source: ICFES. Calculations: Authors. 
Figure 12

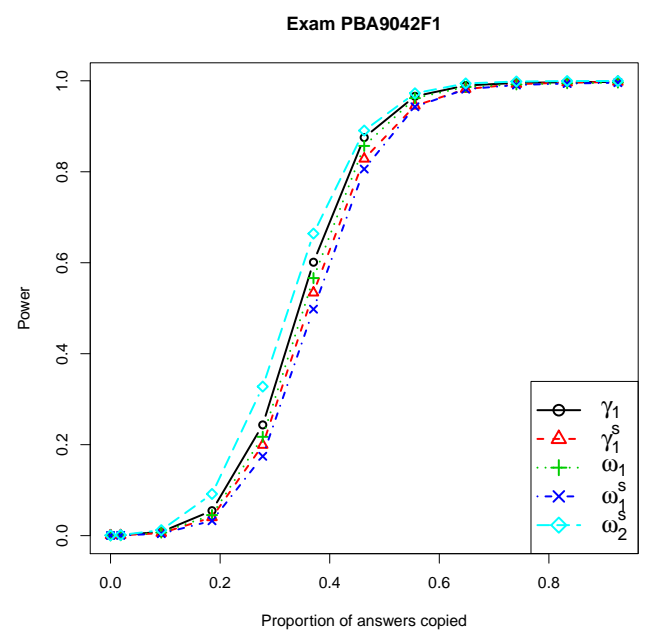

Power in terms of the proportion of answers copied, for all the indices, in the language 9th grade May test. Source: ICFES. Calculations: Authors.

Figure 13

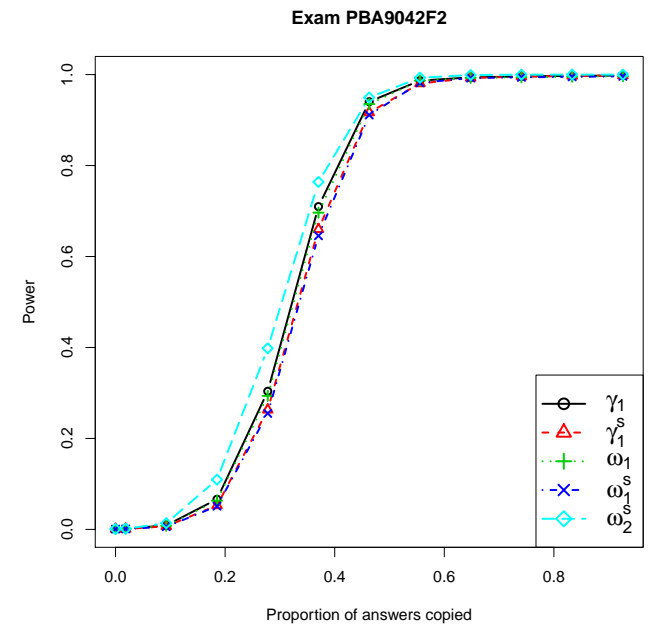

Power in terms of the proportion of answers copied, for all the indices, in the language 9th grade October test. Source: ICFES. Calculations: Authors. 
Figure 14

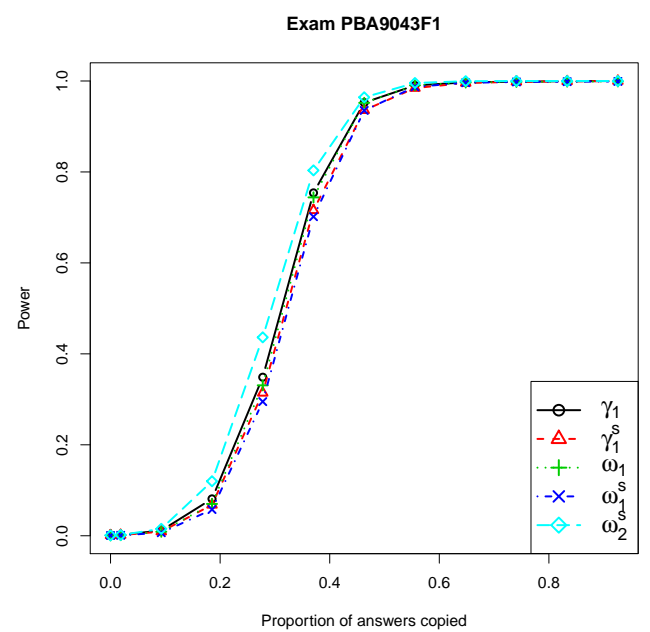

Power in terms of the proportion of answers copied, for all the indices, in the science 9th grade May test. Source: ICFES. Calculations: Authors.

Figure 15

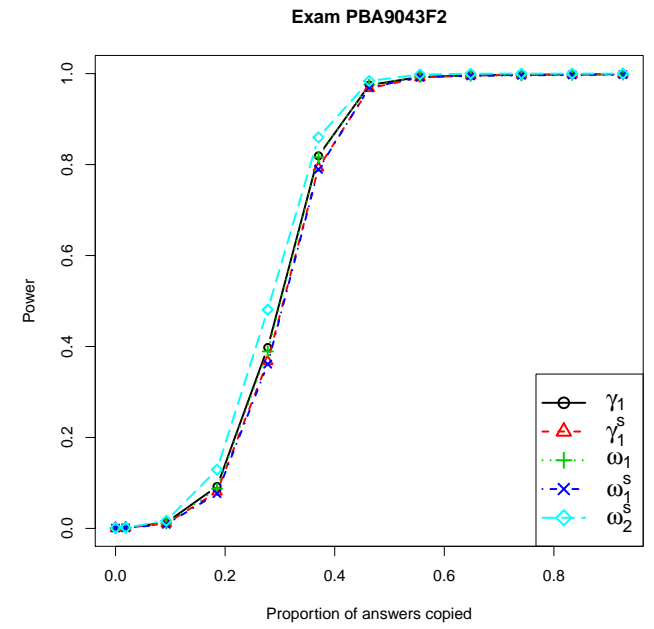

Power in terms of the proportion of answers copied, for all the indices, in the science 9th grade October test. Source: ICFES. Calculations: Authors. 